\title{
Relationship between gut microbiota and lung function decline in patients with chronic obstructive pulmonary disease: a 1-year follow-up study
}

Yu-Chi Chiu ${ }^{1,2,{ }^{\dagger}}$, Shih-Wei Lee ${ }^{1 \dagger}$, Chi-Wei Liu', Tzuo-Yun Lan ${ }^{2 *}$ and Lawrence Shih-Hsin Wu 4, $^{*}$ (D)

\begin{abstract}
Objective: Chronic obstructive pulmonary disease (COPD) is a chronic inflammatory lung disease characterized by a persistent limitation in airflow. Gut microbiota is closely correlated with lung inflammation. However, gut microbiota has not been studied in patients with declining lung function, due to chronic lung disease progression.
\end{abstract}

Subjects and methods: Stool samples were obtained from 55 patients with COPD that were in stable condition at enrolment (stage 1) and at a 1-year follow-up (stage 2). After extracting stool DNA, we performed next generation sequencing to analyse the distribution of gut microbiota.

Results: Patients were divided to control and declining lung function groups, based on whether the rate of forced expiratory volume in $1 \mathrm{~s}\left(\mathrm{FEV}_{1}\right)$ had declined over time. An alpha diversity analysis of initial and follow-up stool samples showed a significant difference in the community richness of microbiota in the declining function group, but not in the control group. At the phylum level, Bacteroidetes was more abundant in the control group and Firmicutes was more abundant in the declining function group. The Alloprevotella genus was more abundant in the control group than in the declining function group. At 1-year follow-up, the mean proportions of Acinetobacter and Stenotrophomonas significantly increased in the control and declining function groups, respectively.

Conclusion: Some community shifts in gut microbiota were associated with lung function decline in COPD patients under regular treatment. Future studies should investigate the mechanism underlying alterations in lung function, due to changes in gut bacterial communities, in COPD.

Keywords: Chronic obstructive pulmonary disease, Stool sample, Next generation sequencing, Gut microbiota, Lung function decline

${ }^{\top}$ Yu-Chi Chiu and Shih-Wei Lee contributed equally to this work

*Correspondence: tylan@ym.edu.tw; Ishwu@hotmail.com

${ }^{2}$ Institute of Public Health, National Yang Ming Chiao Tung University, Taipei, Taiwan

${ }^{4}$ Graduate Institute of Biomedical Sciences, China Medical University, No.

91 Hsueh-Shih Road, Taichung 404, Taiwan

Full list of author information is available at the end of the article

\section{Background}

Chronic obstructive pulmonary disease (COPD) is the fourth leading cause of death worldwide. It affects approximately $10 \%$ of individuals older than 45 years $[1,2]$.This chronic, slowly progressive disease leads to irreversible airway obstruction [3]. COPD is associated with chronic inflammation of the airways and lung parenchyma and also with systemic inflammation [4]. In COPD, chronic inflammation is characterized by 
elevations in alveolar macrophages, neutrophils, T lymphocytes, and innate lymphoid cells, which secrete a variety of proinflammatory mediators, including cytokines, chemokines, growth factors, and lipid mediators [5].

Various inflammatory pathways in lung diseases are regulated by the human microbiota [6]. Previous studies have indicated that gut microbiota stimulated the production of reactive oxygen species in alveolar macrophages [7], which can drive COPD progression [8].

Patients with COPD frequently have other comorbidities, such as cardiovascular disease (CVD) [9], lung cancer [10], diabetes [11], metabolic syndrome [12], malnutrition [13], osteoporosis [14], anxiety, and depression [15]. These comorbidities may influence disease severity and survival. Smoking and aging are well-known risk factors in COPD [16]. Bacterial colonization in the lung has been associated with exacerbations and loss of lung function in patients with COPD $[17,18]$. Moreover, the lung microbiota plays an important role in COPD development $[19,20]$.

Interestingly, studies on ulcerative colitis and Crohn's disease have suggested that the 'gut-lung axis' may be important in COPD pathogenesis [21, 22]. However, the characteristics of gut microbiota in patients with COPD are not fully understood. Previous studies have shown community shifts in gut microbiota, in response to cigarette smoking [23, 24], which suggested that there is some immunological coordination between the lungs and the gut. Despite the importance of gut microbiota in COPD, it remains unknown how gut microbiota might be associated with lung function decline in COPD.

The distributions of respiratory microbiota are significantly different between healthy individuals and individuals with COPD, and between patients with different levels of COPD severity [19]. Recent interest in the influence of probiotics on asthma and COPD [25] has spurred investigations into whether the gut microbiota is related to COPD exacerbation or severity. Indeed, patients with COPD have different faecal microbiota and metabolomes, compared to healthy subjects [26]. Moreover, we previously showed that the gut microbiota was associated with COPD severity [27]. In the present study, we aimed to evaluate changes in gut microbiota during 1 year of lung function decline in patients with COPD who have regular therapy.

\section{Patients and methods}

\section{Patients}

We enrolled 55 patients over 40 years old with COPD. COPD diagnoses and classifications were established according to recommendations from the Global Initiative for Chronic Obstructive Lung Disease (GOLD) [28]. The spirometry tests were conducted by using the Platinum
Elite Series ${ }^{\mathrm{TM}}$ body plethysmograph (MGC Diagnostics Corporation, Saint Paul, MN, USA) according to the recommendations of the European Respiratory Society and the American Thoracic Society [29]. After satisfactory baseline measurements, subjects repeated the spirometry tests $15 \mathrm{~min}$ after bronchodilator inhalation (BEROTEC N $200 \mathrm{mcg}$ ). As aforementioned, spirometry tests after bronchodilator is required to establish a diagnosis of COPD and determine the classification of COPD severity, based on the presence of $\mathrm{FEV}_{1} / \mathrm{FVC}<0.7$ and $\mathrm{FEV}_{1}$ (stage 1: mild $\geq 80 \%$ predicted; stage 2: moderate $50-79 \%$ predicted; stage 3: severe $30-49 \%$ predicted; stage 4: very severe $<30 \%$ predicted). About the smoking situation, 16 (9 in control group and 7 in decline group) of the subjects were current smokers, 33 (15 in control group and 18 in decline group) former smokers and 6 ( 3 in control group and 3 in decline group) never smokers (Table 1). We excluded patients diagnosed with cancer, other immune-related diseases, or viral infections (e.g., hepatitis $B$, hepatitis $C$, human immunodeficiency virus, etc.). A previous study estimated that, over a 3-year period, the forced expiratory volume in $1 \mathrm{~s}\left(\mathrm{FEV}_{1}\right)$ declined by more than $40 \mathrm{ml}$ per year in slightly more than one in three individuals with COPD (38\%) [30]. Based on that finding, we defined lung function decline (the decline group) as an $\mathrm{FEV}_{1}$ decline greater than $40 \mathrm{ml}$ in 1 year. For the control group, we included patients with COPD that showed $\mathrm{FEV}_{1}$ declines less than $40 \mathrm{ml}$ per year and a positive rate of change of $\geq 0 \mathrm{ml}$ per year (i.e., no improvement).

We obtained stool samples from patients with COPD in stable condition (i.e., without exacerbations or the use of antibiotics for any other reason for at least 3 months prior). Samples were acquired at the time of enrolment (first sampling, stage 1) and after a 1-year follow-up (second sampling, stage 2).

\section{Bacterial DNA purification from stool samples}

According to the methods of Chi et al. [27], bacterial DNA was purified from stool samples collected from the 55 subjects using the AllPure Micro Genomic DNA Extraction Kit (Cat. No. ABTGNA022-50, AllBio Science, Inc., Taichung, Taiwan). Briefly, the $0.5 \sim 1 \mathrm{~g}$ stool sample placed in a $15-\mathrm{ml}$ centrifuge tube with $3 \mathrm{ml}$ sterile distilled water and then was mixed thoroughly by vortexing or pipetting. The tubes were centrifuged at $40 \times g$ $(500 \mathrm{rpm})$ for $30 \mathrm{~s}, 362 \times g(1000 \mathrm{rpm})$ for $1 \mathrm{~min}$, and $1449 \times g(3000 \mathrm{rpm})$ for $1 \mathrm{~min}$, respectively. The supernatants were stored at $-20^{\circ} \mathrm{C}$ until DNA extraction.

$1 \mathrm{ml}$ of the supernatant was transferred to a 1.5 microcentrifuge tube and then centrifuged at $13,800 \times g$ $(12,000 \mathrm{rpm})$ for $5 \mathrm{~min}$ and discard the supernatant. The pellet was mixed with $200 \mu \mathrm{l}$ of LB14 buffer and $20 \mu \mathrm{l}$ of Proteinase $\mathrm{K}$ and then incubated at $55{ }^{\circ} \mathrm{C}$ for $30 \mathrm{~min}$ 
Table 1 The clinical characteristics of control and decline groups

\begin{tabular}{|c|c|c|c|}
\hline Variables & Control group & Decline group & $p$ value \\
\hline Male (n) & 27 & 28 & \\
\hline \multicolumn{4}{|l|}{ Age (years) } \\
\hline Mean \pm SD (range) & $70 \pm 10(50-89)$ & $74 \pm 9(51-90)$ & $0.105^{a}$ \\
\hline \multicolumn{4}{|l|}{$\mathrm{BH}(\mathrm{m})$} \\
\hline Mean \pm SD (range) & $1.66 \pm 0.06(1.50-1.77)$ & $1.63 \pm 0.07(1.50-1.77)$ & $0.238^{\mathrm{a}}$ \\
\hline \multicolumn{4}{|l|}{ BW (kg) } \\
\hline Mean \pm SD (range) & $62 \pm 11(43-81)$ & $60 \pm 9(40-75)$ & $0.442^{\mathrm{a}}$ \\
\hline \multicolumn{4}{|l|}{$\mathrm{BMI}$} \\
\hline Mean \pm SD (range) & $22.62 \pm 3.78(14.88-29.72)$ & $22.50 \pm 3.45(15.96-29.14)$ & $0.901^{\mathrm{a}}$ \\
\hline \multicolumn{4}{|l|}{ Smoking (n) } \\
\hline Current smoker & 9 & 7 & $0.777^{c}$ \\
\hline Former smoker & 15 & 18 & \\
\hline Never smoker & 3 & 3 & \\
\hline \multicolumn{4}{|l|}{ WBC (per $\mu l)$} \\
\hline Median (range) & $7060(2580-14,410)$ & $6980(4090-19,590)$ & $0.685^{b}$ \\
\hline \multicolumn{4}{|l|}{ Eosinophil (\%) } \\
\hline Median (range) & $1.9(0.0-36.1)$ & $2.1(0.2-10.9)$ & $0.246^{b}$ \\
\hline \multicolumn{4}{|l|}{ Eosinophil (per $\mu l$ ) } \\
\hline Median (range) & $133(0-4166)$ & $166(19-701)$ & $0.229^{b}$ \\
\hline \multicolumn{4}{|l|}{ Stages (n) } \\
\hline Stage 1 & 8 & 14 & $0.111^{c}$ \\
\hline Stage 2 & 12 & 12 & \\
\hline Stage 3 & 7 & 2 & \\
\hline \multicolumn{4}{|l|}{ CAT } \\
\hline Mean \pm SD & $9.93 \pm 6.68$ & $8.68 \pm 6.02$ & $0.470^{\mathrm{a}}$ \\
\hline Score < 10 (n) & 14 & 19 & $0.226^{c}$ \\
\hline Score $\geqq 10(n)$ & 13 & 9 & \\
\hline \multicolumn{4}{|l|}{ mMRC } \\
\hline Mean \pm SD & $0.96 \pm 0.98$ & $0.89 \pm 1.17$ & $0.810^{\mathrm{a}}$ \\
\hline Score $<2$ (n) & 17 & 21 & $0.334^{c}$ \\
\hline Score $\geqq 2(n)$ & 10 & 7 & \\
\hline \multicolumn{4}{|l|}{$A E(n)$} \\
\hline Yes & 7 & 3 & $0.144^{c}$ \\
\hline No & 20 & 25 & \\
\hline \multicolumn{4}{|l|}{ Medication (n) } \\
\hline LABA & 1 & 2 & $0.175^{c}$ \\
\hline LAMA & 5 & 4 & \\
\hline$L A M A+L A B A$ & 7 & 10 & \\
\hline$I C S+L A B A$ & 7 & 1 & \\
\hline$I C S+L A M A+L A B A$ & 7 & 11 & \\
\hline
\end{tabular}

$S D$ standard deviation, $C O P D$ chronic obstructive pulmonary disease, $n$ number of subjects, $B H$ body height, $W B$ body weight, $B M I$ body mass index, $W B C$ white blood cell, $A E$ acute exacerbation, CAT COPD Assessment Test, $m M R C$ Modified Medical Research Council, FVC forced vital capacity, FEV , forced expiratory volume in one second, LAMA long-acting muscarinic antagonist, LABA long-acting beta agonist, ICS inhaled corticosteroid, ${ }^{\text {T}}$ The statistical analysis was tested by $t$ test; ${ }^{\text {b The statistical }}$

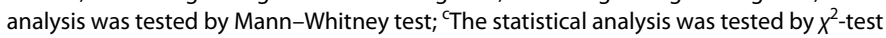

to lyse the pellet. After centrifugation at $13,800 \times g$ $(12,000 \mathrm{rpm})$ for $5 \mathrm{~min}$ at $4{ }^{\circ} \mathrm{C}$, the supernatant was transferred to a new 1.5 microcentrifuge tube and mixed with $20 \mu \mathrm{l}$ of RNaseA and incubated at room temperature for $2 \mathrm{~min}$. After mixing with $500 \mu \mathrm{l}$ of binding buffer, the supernatant was incubated at $55{ }^{\circ} \mathrm{C}$ for $10 \mathrm{~min}$ and then applied to a Genomic Spin Column for DNA purification and washed twice with clean and wash buffers. DNA was eluted from the spin column with $40 \mu \mathrm{l}$ of elution buffer (preheated to $65{ }^{\circ} \mathrm{C}$ ) for a 5 -min incubation 
at room temperature before centrifugation at $13,800 \times g$ $(12,000 \mathrm{rpm})$ for $2 \mathrm{~min}$. The DNA extraction was performed according to the instructions of AllPure Micro Genomic DNA Extraction Kit. The quality of the extracted DNA was visualized by performing $1 \%$ agarose gel electrophoresis and then stored at $-80^{\circ} \mathrm{C}$ until NGS analysis.

\section{MetaV ${ }^{\mathrm{TM}}$ library preparation and illumina MiSeq sequencing}

Next generation sequencing (NGS) library preparations and Illumina MiSeq sequencing were conducted at AllBio Science, Inc. (Taichung, Taiwan). After DNA quantification with a Qubit 2.0 Fluorometer (Invitrogen, Carlsbad, CA, USA), 30-50 ng DNA was used to generate amplicons with a MetaVx ${ }^{\text {TM }}$ Library Preparation kit (GENEWIZ, Inc., South Plainfield, NJ, USA).

The V3-V4 hypervariable regions of prokaryotic $16 \mathrm{~S}$ rDNA were selected for generating amplicon libraries and subsequent taxonomy analyses. The V3-V4 hypervariable regions were amplified with forward primers that contained the sequence "CCTACGGRRBGCASCAGKVRVGAAT" and reverse primers that contained the sequence "GGACTACNVGGGTWTCTAATCC", designed by GENEWIZ. Products from the 1st PCR round were used as templates in a 2nd PCR round for amplicon enrichment. Indexed libraries for downstream NGS sequencing on Illumina Miseq were prepared by adding indexed adapters to the ends of the $16 \mathrm{~S}$ rDNA amplicons.

DNA libraries were validated and quantified with the Agilent 2100 Bioanalyzer (Agilent Technologies, Palo Alto, CA, USA) and the Qubit 2.0 Fluorometer, respectively. According to manufacturer instructions (Illumina, San Diego, CA, USA), the DNA libraries were multiplexed, then loaded on an Illumina MiSeq instrument. DNA libraries were sequenced in the $2 \times 300$ paired-end configuration; image analysis and base calling were performed with MiSeq Control Software provided with the MiSeq instrument.

\section{Data analysis}

16S rRNA data was analysed with the quantitative insights into microbial ecology (QIIME) data analysis package. Forward and reverse reads were joined and assigned to samples, based on barcodes, then the barcode and primer sequences were removed. To improve the quality of the joined sequences, we discarded sequences that did not fulfil the following criteria: sequence length $<200 \mathrm{bp}$, no ambiguous bases, and a mean quality score $\geq 20$. Then, the sequences were compared to the reference database (RDP Gold database). Chimeric sequences were detected and removed with the UCHIME algorithm (https://drive5.com/uchime/uchime_downl oad.html). The clustering program, VSEARCH (1.9.6) [31], was applied to the Silva 119 database, pre-clustered at $97 \%$ sequence identity, to group the effective sequences into operational taxonomic units (OTUs). The Ribosomal Database Program (RDP) classifier was applied with a confidence threshold of 0.8 to assign taxonomic categories to all OTUs. The RDP classifier was applied to the Silva 132 database to assign species-level classifications.

The alpha and beta diversity statistics of amplicon sequencing data were rarefied prior to calculations. In alpha diversity analyses, the Chao1, Shannon, and Simpson indices were calculated in QIIME (version 1.9.1) [32] from rarefied samples. In beta diversity analyses, $\mathrm{R}$ version 3.1.1 (https://cran.r-project.org/bin/windows/base/ old/ 3.1.1/) was used to perform principal component analyses and plots, based on the Brary-Curtis distance matrix. The ecological and heatmap analyses were performed with the pheatmap package (https://cran.r-proje ct.org/src/contrib/Archive/pheatmap/). Differences in taxonomic composition at the genus level were evaluated between different COPD groups with Metastats. Differences in the abundances of microbial communities were evaluated between the groups with strict statistical methods. Significant differences were determined with the multiple hypothesis test and the false discovery rate (FDR) in the rare frequency data. FDR-adjusted p-values were calculated with the Benjamini-Hochberg procedure. Differences in strain abundance between groups were analysed with Statistical Analysis of Metagenomic Profiles (STAMP) software, and Welch's t-test was the default setting for two-group comparisons.

\section{Results}

\section{Demographic and clinical features}

We enrolled 55 patients with COPD that completed a 1-year follow-up (Table 1). Patients were divided into control $(n=27)$ and decline $(n=28)$ groups, based on whether their pre-bronchodilator $\mathrm{FEV}_{1}$ declined less or more than $40 \mathrm{ml} /$ year, respectively. All demographic and clinical features were similar between groups.

The lung function parameters were not significantly different between control and decline groups in stage 1 or stage 2 (Fig. 1), regardless of whether the measurements were performed pre- or post-bronchodilator (Additional file 1: Table S1). However, most lung function parameters changed significantly between stage 1 and stage 2, in both the control and decline groups (Fig. 1). The average parameter values increased in the control group and decreased in the decline group between stages 1 and 2 (Additional file 1: Table S1). In particular, the prebronchodilator $\mathrm{FEV}_{1}$ decreased in the decline group and increased in the control group (Fig. 1). 

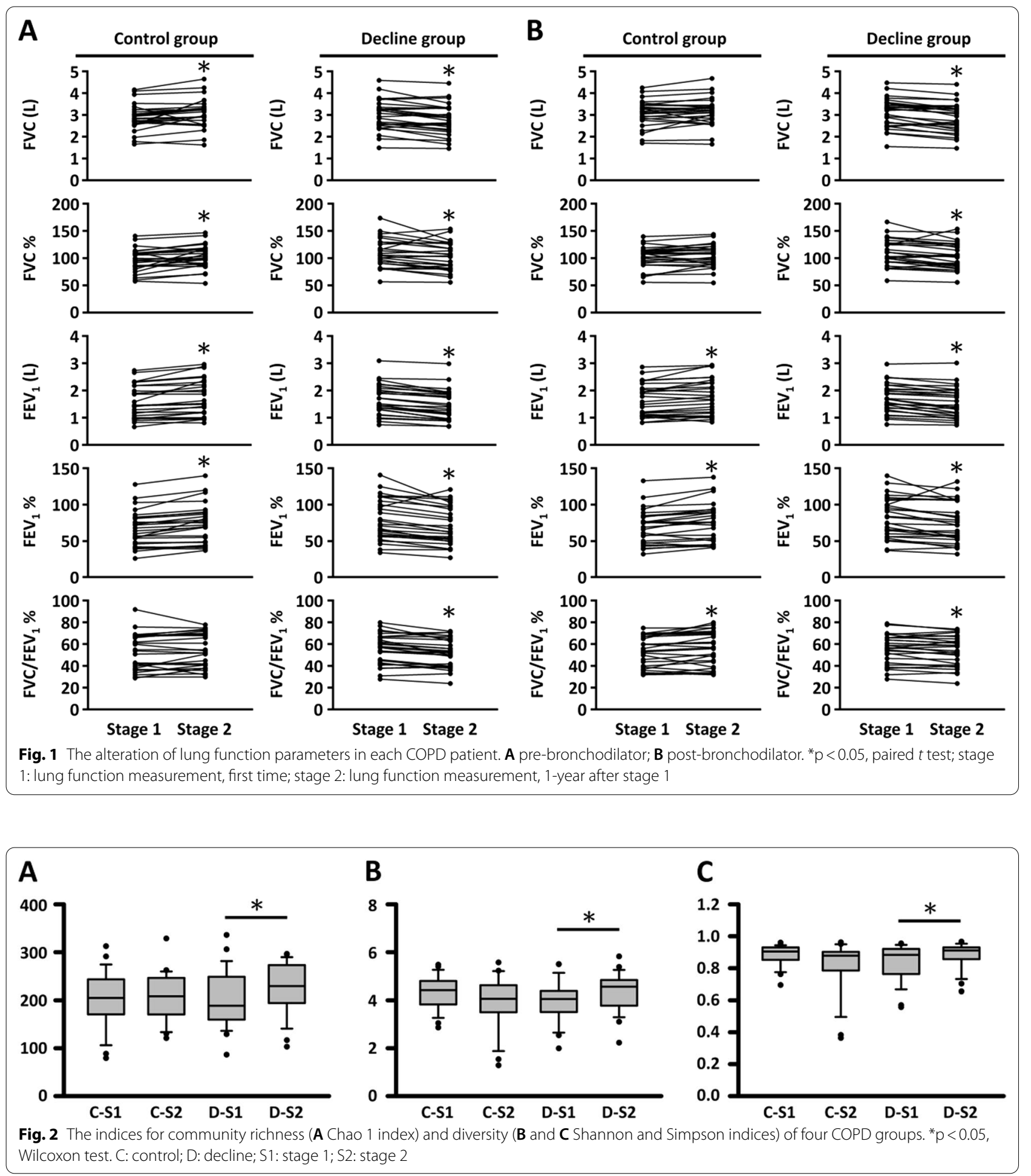

Alterations in alpha and beta diversity

We evaluated the Chao1, Shannon, and Simpson indices to estimate the alpha diversity of gut microbiota in stage 1 and stage 2 samples (Fig. $2 \mathrm{~A}-\mathrm{C}$ ). The OTU richness and diversity indices were not significantly different between the two stages in the control group. However, all three indices increased significantly from stage 1 to stage 2 in the decline group. 
In the beta diversity analysis, we found no clusters in any of these four COPD groups (Additional file 1: Fig. S1).

\section{Taxonomic distributions}

At the phylum level, Bacteroidetes was more abundant in the control group and Firmicutes was more abundant in the decline group (Fig. 3). At the 1-year follow-up, the proportion of Firmicutes decreased in the control group and increased in the decline group (Fig. 3).

At the genus level, the phylogenetic tree of OTUs (Additional file 1: Fig. S2) showed that the 30 most abundant OTUs across all samples belonged to five major phyla (Fig. 3). We plotted heatmaps of the 30 most abundant taxa, identified at the genus or species level, in each study group (Additional file 1: Fig. S3). The group cluster analysis, based on Euclidean distance, showed that the similarity between stages 1 and 2 was higher in the control group than in the decline group. Moreover, the genera in the stage 1 decline group clustered with the genera in the control group (Additional file 1: Fig. S3A). In particular, in the decline group, Tyzzerella 4 was more abundant and Prevotella_9 was less abundant in stage 2 than in stage 1 (Additional file 1: Fig. S3A). In the analysis at the species level (Additional file 1: Fig. S3B), many OTUs were not identified (unclassified).

\section{Differential abundance}

The differential abundance analysis was performed at the genus level. We evaluated the relative abundances of the five genera that showed the largest between-group differences (Table 2). In both stages 1 and 2, Alloprevotella was more abundant in the control group than in the decline group. However, some of the statistical significance might have been due to the contributions of outliers (Additional file 1: Fig. S4C, D).

We compared strain abundances between groups at the genus level (Fig. 4). Significant increases in the mean proportions of Acinetobacter and Stenotrophomonas were observed after 1-year in the control and decline groups, respectively (Fig. 4A, B). At stage 1, the mean proportions were not significantly different between control and decline groups (data not shown). However, at stage 2 , the mean proportions of ten OTUs (genera) were significantly different between control and decline groups (Fig. 4C).

\section{Discussion}

Although COPD is considered a progressive disease, only some patients display an estimated $\mathrm{FEV}_{1}$ decline greater than $40 \mathrm{ml}$ per year [30]. In this study, we found that bacterial richness and diversity in stool samples changed significantly when the $\mathrm{FEV}_{1}$ declined more than $40 \mathrm{ml} /$ year (decline group). Moreover, the most abundant phyla were

\section{A}

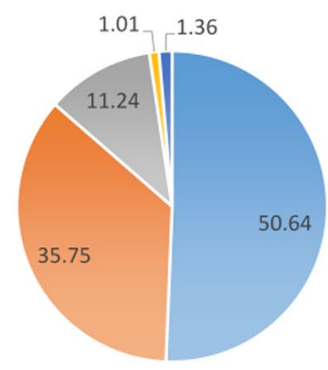

B

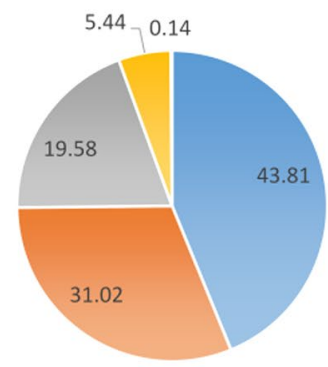

- Bacteroidetes

- Firmicutes

In Proteobacteria

- Fusobacteria

- Verrucomicrobia

\section{Decline-S1 $\mathrm{n}=\mathbf{2 8}$}

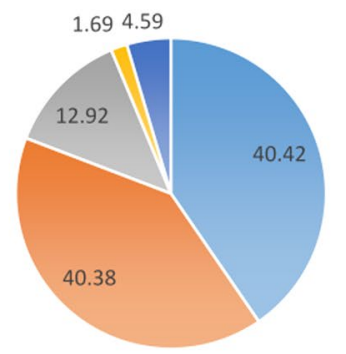

n Bacteroidetes

- Firmicutes

- Proteobacteria

Fusobacteria

- Verrucomicrobia

\section{Decline-S2 $\mathbf{n}=\mathbf{2 8}$}

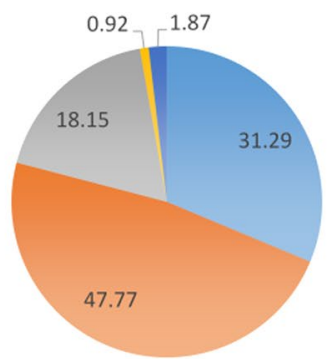

Bacteroidetes

Firmicutes

Proteobacteria

Fusobacteria

- Verrucomicrobia

Fig. 3 The distribution of five most abundant phyla (Firmicutes, Bacteroidetes, Proteobacteria, Fusobacteria, and Verrucomicrobia) in different COPD groups. A: Control-S1; B: Control-S2; C: Decline-S2; D: Decline-S2 
Table 2 The differences in abundance distributions of the five genera with the largest between-group differences

\begin{tabular}{|c|c|c|c|}
\hline Comparison & Phylum & Genus & $\begin{array}{l}\text { Increase }(+) / \\
\text { Decrease } \\
(-)^{\#}\end{array}$ \\
\hline Control-S1 vs & Fusobacteria & Cetobacterium *** & + \\
\hline \multirow[t]{4}{*}{ Control-S2 } & Firmicutes & Lachnospiraceae_NK4A136_group * & - \\
\hline & Proteobacteria & Pluralibacter ${ }^{* * *}$ & - \\
\hline & Firmicutes & Ruminococcaceae_NK4A214_group * & + \\
\hline & Firmicutes & Tyzzerella_4* & - \\
\hline Decline-S1 vs & Proteobacteria & Citrobacter* & + \\
\hline \multirow[t]{4}{*}{ Decline-S2 } & Firmicutes & [Eubacterium]_eligens_group ** & + \\
\hline & Firmicutes & [Eubacterium]_ventriosum_group ${ }^{* *}$ & - \\
\hline & Bacteroidetes & Prevotella_2** & - \\
\hline & Firmicutes & Weissella** & + \\
\hline Decline-S1 vs & Bacteroidetes & Alloprevotella $a^{* * *}$ & + \\
\hline \multirow[t]{4}{*}{ Control-S1 } & Firmicutes & [Eubacterium]_ventriosum_group* & - \\
\hline & Proteobacteria & Pluralibacter ${ }^{* * *}$ & + \\
\hline & Bacteroidetes & Prevotellaceae_NK3B31_group ${ }^{* * *}$ & + \\
\hline & Firmicutes & Ruminiclostridium_6* & + \\
\hline Decline-S2 vs & Firmicutes & Acidaminococcus** & - \\
\hline \multirow[t]{4}{*}{ Control-S2 } & Bacteroidetes & Alloprevotella $a^{* * *}$ & + \\
\hline & Actinobacteria & Bifidobacterium ${ }^{* * *}$ & - \\
\hline & Fusobacteria & Cetobacterium ${ }^{* *}$ & + \\
\hline & Firmicutes & Weissella*** & - \\
\hline
\end{tabular}

\# Reference group: the front group; ${ }^{*} \mathrm{p}<0.05 ;{ }^{* *} \mathrm{p}<0.01 ;{ }^{* * *} \mathrm{p}<0.001$, Fisher's exact test

different between the control (Bacteroidetes) and decline (Firmicutes) groups. Furthermore, both before and after the 1-year follow-up, the Alloprevotella genus was more abundant in the control group than in the decline group. At the 1-year follow-up, the mean proportions of the Acinetobacter and Stenotrophomonas genera significantly increased in the control and decline groups, respectively.

Previous studies have shown that current smoking was strongly associated with the rate of $\mathrm{FEV}_{1}$ decline [30]. Compared to non-smokers, the gut microbiota in current smokers exhibited more abundant Bacteroidetes and less abundant Firmicutes and Proteobacteria [33]. At the genus level, the gut microbiota of smokers harboured more Bacteroides and Prevotella compared to that of non-smokers [34]. Compared to continuing smokers and non-smokers, gut microbiota of ex-smokers that had returned to smoking exhibited more overall microbial diversity and harboured larger proportions of Firmicutes and Actinobacteria, and smaller proportions of Bacteroidetes and Proteobacteria plyla [24]. We found that, among patients with COPD, Bacteroidetes was more abundant when lung function showed little or no decline (control group) and that Firmicutes was more abundant when lung function declined.

In a previous study, $14 \%$ of patients with COPD fulfilled the Rome II criteria for irritable bowel syndrome
(IBS) [35]. Moreover, Mokhlesi et al. found that, among patients with COPD, the prevalence of gastroesophageal reflux symptoms tended to be higher in those with an $\mathrm{FEV}_{1}>50 \%$ of predicted, compared to those with an $\mathrm{FEV}_{1}<50 \%$ of predicted [36]. Furthermore, the coexistence of COPD and small intestinal bacterial overgrowth was associated with changes in microbiota that resembled the instability and reduced diversity frequently observed in post-infectious IBS [37, 38]. In the present study, the decline group showed significant increases in the alpha diversity indices in stage 2 compared to stage 1 . This finding suggested that the gut microbiota was more unstable in the decline group than in the control group. Future studies should investigate potential associations between gastrointestinal disorders, such as IBS and functional dyspepsia, and lung function decline in COPD.

We found a significant increase in the mean proportion of Acinetobacter after 1 year in the control group. A previous study that investigated food introduced in the first year of life and subsequent asthma development showed that unbalanced meat consumption fostered growth of iron scavenging bacteria, such as Acinetobacter, which was related to asthma [39]. However, the biological mechanism remains unknown. It is possible that the increase in Acinetobacter that we observed in the 
A

$\square$ Control-S1 $\square$ Control-S2

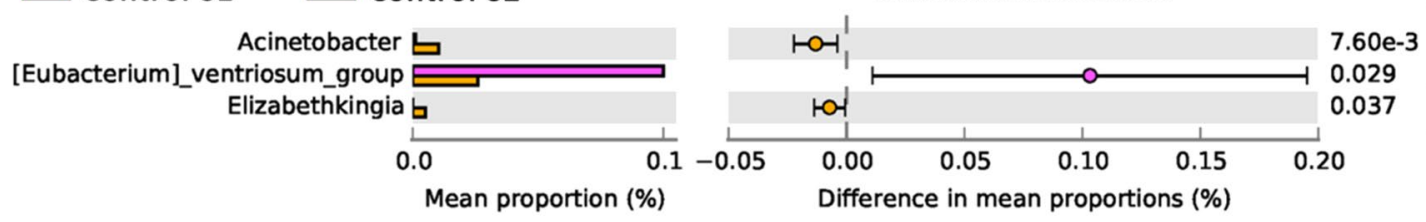

B

$\square$ Decline-S1 صecline-S2

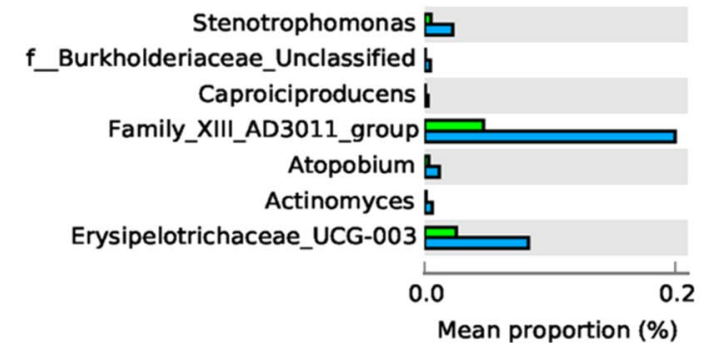

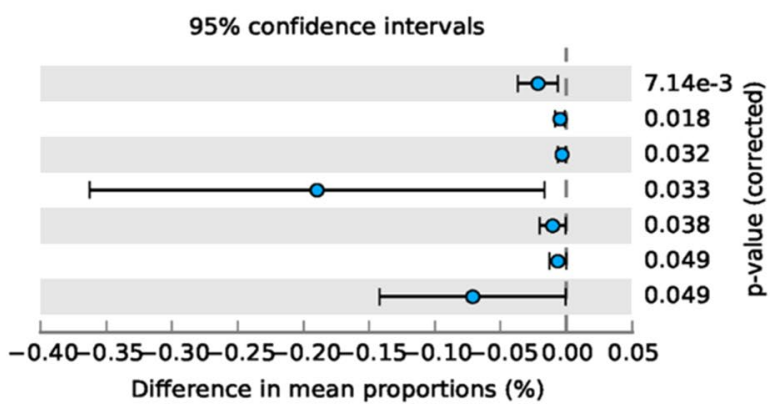

C
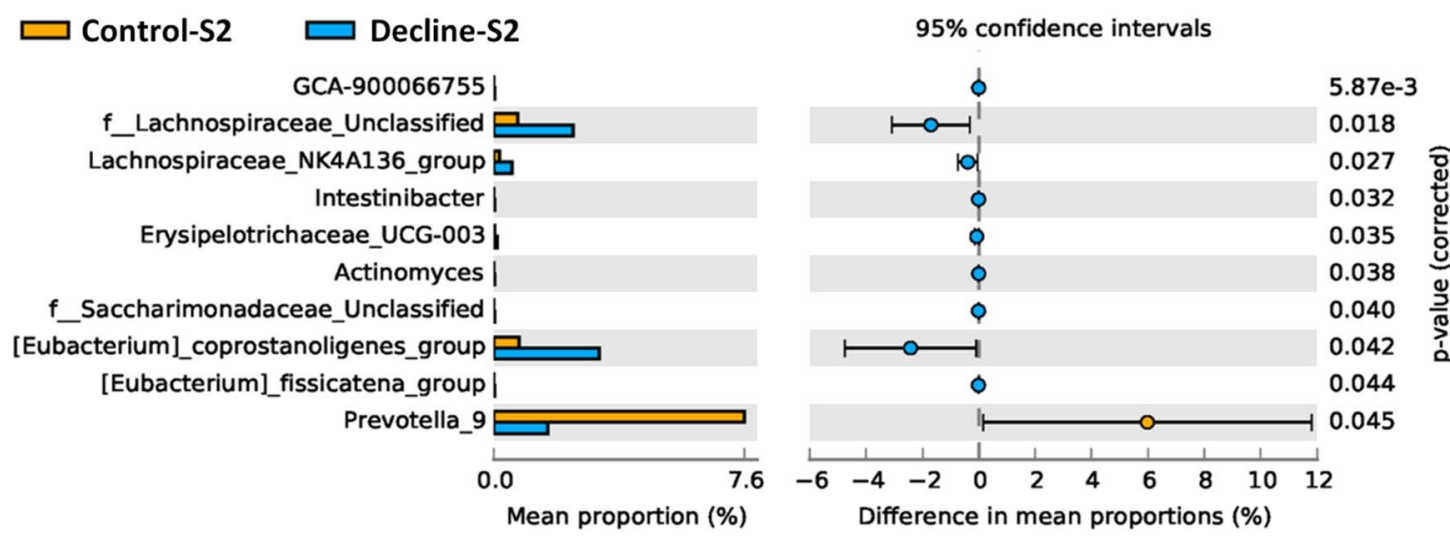

Fig. 4 STAMP analysis at the genus levels showed significant differences in mean proportion between different COPD groups. The statistical analysis was tested by Welch's t-test. A: Control-S1 vs. Control-S2; B: Decline-S1 vs. Decline-S2; C: Control-S2 vs. Decline-S2

control group after 1 year might have been due to food consumption.

In the decline group, the mean proportion of Stenotrophomonas significantly increased after 1 year. Stenotrophomonas maltophilia colonies are commonly found in the respiratory tracts of patients with chronic lung disease [40]. Indeed, some community-acquired pneumonia cases are caused by Gram-negative bacteria, particularly Pseudomonas aeruginosa, Klebsiella pneumoniae, Acinetobacter baumannii, and Stenotrophomonas maltophilia [41, 42]. However, we did not detect Acinetobacter baumannii or Stenotrophomonas maltophilia in stool samples from patients with COPD. Furthermore, it remains unknown whether increases in the proportions of Acinetobacter and Stenotrophomonas in gut are related to COPD progression.

We also found a reduction in the relative abundance of Prevotella_2 after 1 year in the decline group (Table 2). Moreover, at stage 2, the control group had a higher mean proportion of Prevotella_9 than the decline group (Fig. 4). A previous study showed an association between lung microbiota and COPD severity (defined by computed tomography). They found that patients with mild COPD had higher abundances of Prevotella in lung 
samples than patients with severe COPD [43]. In another study, LPS/elastase-treated mice had reduced representation of Prevotella in the lung [44]. In gut, Prevotella strains are associated with plant-rich diets, but they are also linked with chronic inflammatory conditions [45]. Based on those findings, we suggest that the abundance of Prevotella might be associated with a putative COPD subtype that is related to the rate of lung function decline.

The microbiota could be used as potential markers for the progression of HBV-related acute-on-chronic liver failure [46] and the management of cancer [47]. Previous studies indicated that gut microbiota regulated lung inflammation [48] and was associated with respiratory diseases [49]. In addition, in mice model, gut microbiota from patients with COPD induced lung inflammation and adaptive immune responses [50]. The increased ratio of Firmicutes/Bacteroidetes species as well as segmented filamentous bacteria colonization is associated with lung IL-17 and IL-22 responses and enhances airway hyperresponsiveness [48]. The increased Bacteroidetes and Actinobacteria species decrease airway inflammation by the production of short chain fatty acids and regulation of Treg cells [48]. Whether any immune molecules and cells are involved in the lung function decline caused by the intestinal microbiota needs further investigation.

Our study had some limitations. First, we had relatively small COPD groups, which could have limited our statistical power. Future studies are necessary, with larger groups, to confirm our findings. Second, we mostly analysed microbiota genera, rather than species. Therefore, we could not infer the roles of certain species, particularly pathogens, in COPD progression. Finally, in this study, we chosen the definition of decline group ( $\mathrm{FEV}_{1}$ declined more than $40 \mathrm{ml} /$ year) according to previous study [30] and because the annualized absolute loss of $\mathrm{FEV}_{1}$ of $\geqq 40 \mathrm{ml} /$ year was a significant predictor for incident COPD risk among ever smokers without prevalent obstructive and nonobstructive lung disease at baseline examination [51]. However, a recent report indicated that year-to-year changes in $\mathrm{FEV}_{1}$ should exceed $15 \%$ before considering it as a clinically meaningful change in patients with COPD [52]. In this study, 11 of decline subjects were fit the criteria year-to-year $\mathrm{FEV}_{1}$ variability exceeded $15 \%$. In future study, it would be worthwhile to evaluate the difference in gut microbiota between subjects whose losses year-to-year $\mathrm{FEV}_{1}$ exceed $40 \mathrm{ml}$ and $15 \%$.

In conclusion, we found that community shifts in gut microbiota were associated with lung function decline. Our results suggested that the gut microbiota might influence COPD progression.

\section{Abbreviations}

SD: Standard deviation; COPD: Chronic obstructive pulmonary disease; $\mathrm{BH}$ : Body height; WB: Body weight; BMI: Body mass index; WBC: White blood cell; AE: Acute exacerbation; CAT: COPD assessment test; mMRC: Modified Medical Research Council; FVC: Forced vital capacity; FEV 1 : Forced expiratory volume in one second; LAMA: Long-acting muscarinic antagonist; LABA: Long-acting beta agonist; ICS: Inhaled corticosteroid; OTU: Operational taxonomic unit; GOLD: Global initiative for chronic obstructive lung disease; NGS: Next generation sequencing; QIIME: Quantitative insights into microbial ecology; RDP: Ribosomal database program; FDR: False discovery rate; STAMP: Statistical analysis of metagenomic profiles.

\section{Supplementary Information}

The online version contains supplementary material available at https://doi. org/10.1186/s12931-022-01928-8.

Additional file 1. Supplemental Table 1. Comparison of lung function parameters for control and decline groups. Supplemental Fig. 1. Plot of principal component analysis (PCA) for different COPD groups. S1: stage 1; S2: stage 2. Supplemental Fig. 2. The phylogenic tree of the 30 most abundant OTUs across the samples at the genus level. Supplemental Fig. 3. The heatmap analysis of the top 30 OTUs. A: At genus level. B: At species level.

\section{Authors' contributions}

LSHW and TYL conceived and designed the study. SWL, CWL, and LSHW acquired the data. YCC, CWL, and LSHW analyzed and interpreted the data. YCC, SWL, CWL, TYL, and LSHW drafted or revised the article. YCC, SWL, CWL, TYL, and LSHW approved the final manuscript. All authors read and approved the final manuscript.

\section{Funding}

This work was supported by Taoyuan General Hospital, Ministry of Health and Welfare, Taoyuan, Taiwan (PTH108003 to SWL) and research grant (1 JA8) from Center for Allergy, Immunology, Microbiome (A.I.M.), China Medical University Hospital, Taichung, Taiwan.

\section{Availability of data and materials}

The data of $16 \mathrm{~S}$ rRNA V3 + V4 hypervariable regions by sequencing for stool from COPD patients had submitted to European Nucleotide Archive, Accession No: PRJEB43280.

\section{Declarations}

\section{Ethics approval and consent to participate}

The Ethics Committee of Taoyuan General Hospital (Taoyuan, Taiwan) IRB approved the research protocol (IRB No.: TYGH107039). All subjects enrolled in the study provided written informed consent.

\section{Consent for publication}

Not applicable.

\section{Competing interests}

The authors declare no conflicts of interest.

\section{Author details}

${ }^{1}$ Department of Internal Medicine, Taoyuan General Hospital, Ministry of Health and Welfare, Taoyuan, Taiwan. ${ }^{2}$ Institute of Public Health, National Yang Ming Chiao Tung University, Taipei, Taiwan. ${ }^{3}$ Department of Nursing, Yuanpei University of Medical Technology, Hsinchu, Taiwan. ${ }^{4}$ Graduate Institute of Biomedical Sciences, China Medical University, No. 91 Hsueh-Shih Road, Taichung 404, Taiwan. ${ }^{5}$ Center of Allergy, Immunology, and Microbiome (A.I.M.), China Medical University Children's Hospital, Taichung, Taiwan. 
Received: 8 September 2021 Accepted: 5 January 2022

Published: 15 January 2022

\section{References}

1. Berg K, Wright JL. The pathology of chronic obstructive pulmonary disease: progress in the 20th and 21st centuries. Arch Pathol Lab Med. 2016:140:1423-8.

2. Lozano R, Naghavi M, Foreman K, Lim S, Shibuya K, Aboyans V, et al. Global and regional mortality from 235 causes of death for 20 age groups in 1990 and 2010: a systematic analysis for the Global Burden of Disease Study 2010. Lancet. 2012:380:2095-128.

3. Pauwels RA, Rabe KF. Burden and clinical features of chronic obstructive pulmonary disease (COPD). Lancet. 2004;364:613-20.

4. Barnes PJ. Cellular and molecular mechanisms of chronic obstructive pulmonary disease. Clin Chest Med. 2014;35:71-86.

5. Barnes PJ. Inflammatory mechanisms in patients with chronic obstructive pulmonary disease. J Allergy Clin Immunol. 2016;138:16-27.

6. Shukla SD, Budden KF, Neal R, Hansbro PM. Microbiome effects on immunity, health and disease in the lung. Clin Transl Immunol. 2017;6:e133.

7. Clarke TB. Early innate immunity to bacterial infection in the lung is regulated systemically by the commensal microbiota via nod-like receptor ligands. Infect Immun. 2014:82:4596-606.

8. Domej W, Oettl K, Renner W. Oxidative stress and free radicals in COPDimplications and relevance for treatment. Int J Chron Obstruct Pulmon Dis. 2014;9:1207-24.

9. Lahousse L, Tiemeier H, Ikram MA, Brusselle GG. Chronic obstructive pulmonary disease and cerebrovascular disease: a comprehensive review. Respir Med. 2015;109:1371-80.

10. Durham AL, Adcock IM. The relationship between COPD and lung cancer. Lung Cancer. 2015;90:121-7.

11. Gläser S, Krüger S, Merkel M, Bramlage P, Herth FJ. Chronic obstructive pulmonary disease and diabetes mellitus: a systematic review of the literature. Respiration. 2015:89:253-64.

12. Clini E, Crisafulli E, Radaeli A, Malerba M. COPD and the metabolic syndrome: an intriguing association. Intern Emerg Med. 2013;8:283-9.

13. Gea J, Martínez-Llorens J, Barreiro E. Nutritional abnormalities in chronic obstructive pulmonary disease. Med Clin. 2014;143:78-84.

14. Sarkar M, Bhardwaj R, Madabhavi I, Khatana J. Osteoporosis in chronic obstructive pulmonary disease. Clin Med Insights Circ Respir Pulm Med. 2015;9:5-21.

15. Pumar MI, Gray CR, Walsh JR, Yang IA, Rolls TA, Ward DL. Anxiety and depression-Important psychological comorbidities of COPD. J Thorac Dis. 2014;6:1615-31.

16. Takahashi S, Betsuyaku T. The chronic obstructive pulmonary disease comorbidity spectrum in Japan differs from that in western countries. Respir Investig. 2015;53:259-70.

17. Wang Z, Bafadhel M, Haldar K, Spivak A, Mayhew D, Miller BE, et al. Lung microbiota dynamics in COPD exacerbations. Eur Respir J. 2016:47:1082-92.

18. Miravitlles M, Anzueto A. Role of infection in exacerbations of chronic obstructive pulmonary disease. Curr Opin Pulm Med. 2015;21:278-83.

19. Mammen MJ, Sethi S. COPD and the microbiome. Respirology. 2016;21:590-9.

20. Pragman AA, Kim HB, Reilly CS, Wendt C, Isaacson RE. The lung microbiota in moderate and severe chronic obstructive pulmonary disease. PLoS ONE. 2012;7:e47305.

21. Budden KF, Gellatly SL, Wood DL, Cooper MA, Morrison M, Hugenholtz P, et al. Emerging pathogenic links between microbiota and the gut-lung axis. Nat Rev Microbiol. 2016;15:55-63.

22. Ekbom A, Brandt L, Granath F, Löfdahl CG, Egesten A. Increased risk of both ulcerative colitis and Crohn's disease in a population suffering from COPD. Lung. 2008;186:167-72.

23. Allais L, Kerckhof FM, Verschuere S, Bracke KR, De Smet R, Laukens D, et al. Chronic cigarette smoke exposure induces microbial and inflammatory shifts and mucin changes in the murine gut. Environ Microbiol. 2016:18:1352-63

24. Biedermann L, Zeitz J, Mwinyi J, Sutter-Minder E, Rehman A, Ott SJ, et al. Smoking cessation induces profound changes in the composition of the intestinal microbiota in humans. PLoS ONE. 2013;8:e59260.
25. Anand S, Mande SS. Diet, microbiota and gut-lung connection. Front Microbiol. 2018;9:2147.

26. Bowerman KL, Rehman SF, Vaughan A, Lachner N, Budden KF, Kim RY, et al. Disease-associated gut microbiome and metabolome changes in patients with chronic obstructive pulmonary disease. Nat Commun. 2020;11:5886

27. Chiu YC, Lee SW, Liu CW, Lin RC, Huang YC, Lan TY, et al. Comprehensive profiling of the gut microbiota in patients with chronic obstructive pulmonary disease of varying severity. PLOS ONE. 2021:16:e0249944.

28. Global Initiative for Chronic Obstructive Lung Disease. Global Strategy for the Diagnosis, Management, and Prevention of Chronic Obstructive Pulmonary Disease. 2019. https://goldcopd.org/wp-content/uploads/2018/ 11/GOLD-2019-v1.7-FINAL-14Nov2018-WMS.pdf Accessed: December 16, 2020.

29. Miller MR, Hankinson J, Brusasco V, Burgos F, Casaburi R, Coates A, et al. Standardisation of spirometry. Eur Respir J. 2005;26:319-38.

30. Vestbo J, Edwards LD, Scanlon PD, Yates JC, Agusti A, Bakke P, et al. Changes in forced expiratory volume in 1 second over time in COPD. N Engl J Med. 2011;365:1184-92.

31. Rognes T, Mahe' F, Flouri T, McDonald D, Schloss P. Woodcroft BJ. vsearch: VSEARCH 1.9.6 (Version v1.9.6). Zenodo, 2016. https://doi.org/10.5281/ zenodo.44512.

32. Caporaso JG, Kuczynski J, Stombaugh J, Bittinger K, Bushman FD, Costello EK, et al. QIIME allows analysis of high-throughput community sequencing data. Nat Methods. 2010;7:335-6.

33. Lee SH, Yun Y, Kim SJ, Lee EJ, Chang Y, Ryu S, et al. Association between cigarette smoking status and composition of gut microbiota: populationbased cross-sectional study. J Clin Med. 2018;7:282.

34. Benjamin JL, Hedin CR, Koutsoumpas A, Ng SC, McCarthy NE, Prescott $\mathrm{NJ}$, et al. Smokers with active Crohn's disease have a clinically relevant dysbiosis of the gastrointestinal microbiota. Inflamm Bowel Dis. 2012;18:1092-100.

35. Niklasson A, Strid H, Simrén M, Engström CP, Björnsson E. Prevalence of gastrointestinal symptoms in patients with chronic obstructive pulmonary disease. Eur J Gastroenterol Hepatol. 2008;20:335-41.

36. Mokhlesi B, Morris AL, Huang CF, Curcio AJ, Barrett TA, Kamp DW. Increased prevalence of gastroesophageal reflux symptoms in patients with COPD. Chest. 2001:119:1043-8.

37. Schwille-Kiuntke J, Mazurak N, Enck P. Systematic review with meta-analysis: post-infectious irritable bowel syndrome after travellers' diarrhoea. Aliment Pharmacol Ther. 2015;41:1029-37.

38. Mooney PD, Hayman J, Pickering G, Sanders DS. Are we underestimating the prevalence of small intestinal bacterial overgrowth in irritable bowel syndrome? Neurogastroenterol Motil. 2015;27:1190-1.

39. Hose AJ, Pagani G, Karvonen AM, Kirjavainen PV, Roduit C, Genuneit J, et al. Excessive unbalanced meat consumption in the first year of life increases asthma risk in the PASTURE and LUKAS2 birth cohorts. Front Immunol. 2021;12:651709.

40. Pathmanathan A, Waterer GW. Significance of positive Stenotrophomonas maltophilia culture in acute respiratory tract infection. Eur Respir J. 2005:25:911-4.

41. Aliberti S, Cilloniz C, Chalmers JD, Zanaboni AM, Cosentini R, Tarsia P, et al. Multidrug-resistant pathogens in hospitalised patients coming from the community with pneumonia: a European perspective. Thorax 2013:68:997-9.

42. Cillóniz C, Ewig S, Polverino E, Marcos MA, Esquinas C, Gabarrús A, et al. Microbial aetiology of community acquired pneumonia and its relation to severity. Thorax. 2011;66:340-6.

43. Engel M, Endesfelder D, Schloter-Hai B, Kublik S, Granitsiotis MS, Boschetto $\mathrm{P}$, et al. Influence of lung $\mathrm{CT}$ changes in chronic obstructive pulmonary disease (COPD) on the human lung microbiome. PLOS ONE. 2017:12:e0180859.

44. Yadava K, Pattaroni C, Sichelstiel AK, Trompette A, Gollwitzer ES, Salami $\mathrm{O}$, et al. Microbiota promotes chronic pulmonary inflammation by enhancing IL-17A and autoantibodies. Am J Respir Crit Care Med. 2016;193:975-87.

45. Ley RE. Gut microbiota in 2015: prevotella in the gut: choose carefully. Nat Rev Gastroenterol Hepatol. 2016;13:69-70.

46. Wang $\mathrm{K}$, Zhang Z, Mo ZS, Yang XH, Lin BL, Peng L, et al. Gut microbiota as prognosis markers for patients with HBV-related acute-on-chronic liver failure. Gut Microbes. 2021:13:1-15. 
47. Veziant J, Villéger R, Barnich N, Bonnet M. Gut microbiota as potential biomarker and/or therapeutic target to improve the management of cancer: focus on colibactin-producing Escherichia coli in colorectal cancer. Cancers (Basel). 2021;13:2215.

48. McAleer JP, Kolls JK. Contributions of the intestinal microbiome in lung immunity. Eur J Immunol. 2018;48:39-49.

49. Chunxi L, Haiyue L, Yanxia L, Jianbing P, Jin S. The gut microbiota and respiratory diseases: new evidence. J Immunol Res. 2020;2020:2340670.

50. Li N, Dai Z, Wang Z, Deng Z, Zhang J, Pu J, et al. Gut microbiota dysbiosis contributes to the development of chronic obstructive pulmonary disease. Respir Res. 2021;22:274.

51. Petersen H, Sood A, Meek PM, Shen X, Cheng Y, Belinsky SA, et al. Rapid lung function decline in smokers is a risk factor for COPD and is attenuated by angiotensin-converting enzyme inhibitor use. Chest. 2014;145:695-703.

52. Crim C, Frith LJ, Midwinter D, Donohue JF. FEV1 minimum important difference versus minimal detectable difference? In search of the unicorn. Am J Respir Crit Care Med. 2021;203:1573-6.

\section{Publisher's Note}

Springer Nature remains neutral with regard to jurisdictional claims in published maps and institutional affiliations.

- fast, convenient online submission

- thorough peer review by experienced researchers in your field

- rapid publication on acceptance

- support for research data, including large and complex data types

- gold Open Access which fosters wider collaboration and increased citations

- maximum visibility for your research: over $100 \mathrm{M}$ website views per year

At BMC, research is always in progress.

Learn more biomedcentral.com/submissions 